\title{
Positron Annihilation Lifetime Spectroscopy and Dielectric Measurements of Natural Kaolinite and Kaolinite Intercalated by Potassium Acetate
}

\author{
K. $\operatorname{LeluK}^{a, *}, \mathrm{~K} . \mathrm{OrzeChOWSKI}^{a}, \mathrm{~K} . \mathrm{JerIE}^{b}, \mathrm{~A} . \mathrm{BARANOWSKI}^{b}$, \\ T. SŁONKA ${ }^{c}$ AND J. GŁOWIŃSKI ${ }^{c}$ \\ ${ }^{a}$ Faculty of Chemistry, Wrocław University \\ F. Joliot-Curie 14, 50-383 Wrocław, Poland \\ ${ }^{b}$ Institute of Experimental Physics, University of Wrocław \\ pl. M. Borna 9, 50-204 Wrocław, Poland \\ ${ }^{c}$ Faculty of Chemistry, Wrocław University of Technology \\ Wybrzeże Wyspiańskiego 27, 50-370 Wrocław, Poland
}

\begin{abstract}
Intercalation of clay minerals consists in inserting of guest molecules into interlayer area. It results in expanding the interlayer distance and changes of physical and chemical properties of the material. Dielectric spectroscopy, positron annihilation lifetime experiments, X-ray, and thermoanalysis were jointly applied to investigate the structural changes accompanying intercalation.
\end{abstract}

PACS numbers: 91.60.-x, 77.22.-d, 78.70.Bj

\section{Introduction}

Clays are well-known raw materials used for fabricating ceramics and crockery [1]. Very important for technology but also for pure science is their ability to adsorption, exfoliation, and intercalation resulting in new, interesting nanomaterials.

Kaolinite is a bilayered aluminosilicate, where gibbsite to silicate ratio is $1: 1$. Kaolinite does not swell in water, because the interlayer interactions are too strong to be destroyed by water molecules penetrating between. However,

${ }^{*}$ corresponding author; e-mail: leluk@wcheto.chem.uni.wroc.pl 
in concentrated solutions of some inorganic salts [2] and strongly polar organic compounds, kaolinite swells and this process is assisted with intercalation of guest molecules into interlayer area. Intercalation expands the interlayer spacing from $7.14 \AA$ for natural kaolinite to the higher value characteristic of the intercalating guest molecule. Intercalation alternates most of the material properties and many experimental techniques may characterize it.

In this paper the dielectric and positron annihilation data will be interpreted simultaneously with X-ray diffraction (XRD), and thermogravimetric (TG) experiments. The application of different but complementary methods will give a precise understanding of molecular properties of investigated materials. Positron annihilation is suitable for detection of free volumes, dielectric spectroscopy allows one to investigate molecular movement and dynamics of structural changes.

In most solids, dielectric permittivity $\left(\varepsilon^{\prime}\right)$ is low and close to the square of refractive index $(n)$. However, in some materials, as in crystals with rotational phase, in glasses [3], in ice and ice clathrates [4] a considerable increase in permittivity is observed. The increase demonstrates a possibility of some movement - librations or even reorientation of polar molecule or polar groups. This phenomenon could be observed when free volume for the movement is available and polar molecules are not firmly bounded to th surroundings. The existence of free volumes, crystal imperfections, and empty areas can be confirmed by positron annihilation lifetime spectroscopy (PALS).

PALS consists in measurements of a lifetime of positrons emitted by radioactive source. Positrons injected to the investigated material have to be thermalized and after that they can annihilate. Most of positrons rapidly annihilate with free electrons. The lifetime of this process is of order of $0.18 \mathrm{~ns}$ and strongly depends on electron density in the material [5]. In imperfect crystals or grained materials some of positrons could reach a grain boundary or a boundary of larger vacancies. Lifetime of such positrons could increase even twice. When sufficiently large, empty volumes exist in the investigated material, some positrons can form pairs constituted of electron and positron [5]. Such pairs, named "positronium" (Ps) are formed in two states: ortho-positronium (o-Ps) with parallel spins and parapositronium ( $p$-Ps) with anti-parallel spins. The ratio of population of $o$-Ps to $p$-Ps is $3: 1$ in room temperature. Para-positronium is a less stable particle. In vacuum its lifetime is of order of $0.12 \mathrm{~ns}$ [6]. Ortho-positronium can live in vacuum even $142 \mathrm{~ns}[6,7]$. In condensed matter, the lifetime of $o$-Ps is considerably shortened. It can exist in free volumes with low electron density only. The relationship between lifetime and free volume is the basis application of PALS for investigation of micro-porosity of solids [8, 9] and liquids [10].

\section{Experimental}

Kaolinite was obtained from Maria deposit, Lower Silesia, Poland. The material was washed by water and centrifuged. To prepare intercalated material, 
kaolinite was shaken with aqueous solution of potassium acetate for 36 hours. The suspension was left for 14 days and then centrifuged. Before physicochemical experiments, the samples of natural kaolinite and intercalated kaolinite were kept over $\mathrm{P}_{2} \mathrm{O}_{5}$ at least for 14 days.

XRD experiments were made by a DRON-2 powder diffractometer. Co $K_{\alpha}$ lamp was used as a source of radiation. The intensity of (001) line was measured in $0.05^{\circ}$ steps. The (001) reflection was approximated to parabolic function and the maximum was used for calculation of spacing of the layers.

TG measurements were made by Derivatograph SETSYS 16/18 (SETARAM, France). Temperature was increasing with the rate of $5^{\circ} \mathrm{C} / \mathrm{min}$. All data were collected by a computer system.

PALS measurements were performed at room temperature. The samples were prepared as pellets formed inside an aluminum ring of diameter of $25 \mathrm{~mm}$ and thickness of $2 \mathrm{~mm}$. Between such two pellets of the investigated material, the radioactive source $\left({ }^{22} \mathrm{NaCl}\right)$ covered by Hostaphan ${ }^{\circledR}$ foil was placed. For registering the spectra, fast coincidence techniques were used. The equipment is based on Burle 8850 photomultipliers and conical plastic scintillators. Ortec 583 constant fraction discriminators and Ortec 457 time to pulse height converter were employed. The time resolution was $270 \mathrm{ps}$, the channel width $51 \mathrm{ps} / \mathrm{ch}$. Zero time stability was better than 0.5 ps per 24 hours. The spectrum was analyzed using POSITRONFIT program [11]. All the spectra was resolved into three components. Dielectric experiments were performed in three frequency ranges: $0.1 \mathrm{kHz}-1 \mathrm{MHz}$ using an HP 4284A RLC bridge, $1 \mathrm{MHz}-1 \mathrm{GHz}$ using an HP 4191A Impedance Analyzer, 2.6-4.0 GHz using a wave-guide equipment [12]. In the frequency interval $1-4 \mathrm{GHz}$ the lumped capacitance method was used [12]. Air and teflon ${ }^{\circledR}$ were used as standards in calibration procedure in all the frequency ranges. The sample holder filled by the powdered sample was mounted on the inner end of a coaxial wave-guide. The fraction of the capacitor volume filled by the sample was estimated from the mass of the probe and density of the material. Details of construction of the sample holder and handling in filling by the sample was described before [2].

\section{Results and discussion}

The XRD spectra obtained for pure kaolinite and its intercalated derivative at room temperature are shown in Fig. 1. In natural kaolinite the peak at $2 \theta=14.5^{\circ}$ is related to the interlayer distance $7.1 \AA$. After intercalation the peak characteristic of unmodified kaolinite is still observable, but a new one, high intensity peak at $2 \theta=7.3^{\circ}$ related to the expanded layers (the distance $14.1 \AA$ ) is observed. Spacings in natural kaolinite and in kaolinite intercalated by potassium acetate are similar to those obtained by other authors $[2,13,14]$. Using the obtained data it is possible to estimate the thickness of free part of interlayer space. Assuming that the thickness of aluminosilicate layer is $5.3 \AA$, the interlayer 
distance for kaolinite is approximately $1.8 \AA$, and for intercalate approximately $8.8 \AA[15]$. The comparison of intensities of peaks at $7.3^{\circ}$ and $14.5^{\circ}$ allows one to estimate the efficiency of intercalation as $90 \%$.

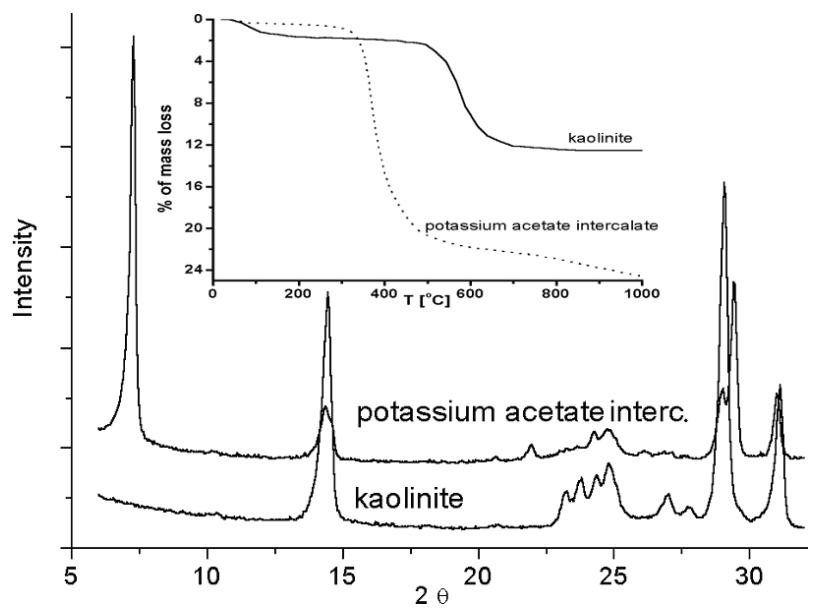

Fig. 1. XRD results obtained for kaolinite and its intercalated derivative at room temperature. Insetted picture depicts TG curves obtained for pure kaolinite and its intercalate.

The understanding of properties of the investigated material requires information related to the degree of filling of the interlayer area by guest molecules. Unfortunately, the intercalate is not a stoichiometric compound and this property is impossible to be predicted. However, thermogravimetric analysis allows one to estimate it. The insetted picture in Fig. 1 presents the thermogravimetric curve in pure kaolinite and in the intercalated material. There are two regions where the decrease of mass are observed. The first one, a small decrease between $50-100^{\circ} \mathrm{C}$, is probably related to removing of water adsorbed at the surface of the material. The decrease is similar for kaolinite and the intercalate. The second process, occurring between 300 and $400^{\circ} \mathrm{C}$ in the case of pure kaolinite leads to $10 \%$ decrease in mass of the sample and is related to dehydroxylation of the material. In the case of intercalated kaolinite the decrease is much larger (18.5\%) and should be interpreted as simultaneous removing of intercalated potassium acetate and dehydroxylation.

The comparison of TG curves in kaolinite and intercalate allows one to calculate the mass fraction of potassium acetate in intercalated material:

$$
w=\frac{W_{2}-W_{1}}{100-W_{1}}
$$

where $w$ is the mass fraction of potassium acetate, $W_{1}$ is the percentage decrease in mass of kaolinite in dehydratation process, $W_{2}$ is the percentage decrease in mass of intercalate in simultaneous removing of potassium acetate and dehydratation. 
Following that, the mass fraction of potassium acetate in the investigated material is estimated as 0.094. Taking into account that the elementary cell of kaolinite contains two structural elements it is possible to calculate that a single potassium acetate molecule falls into 2 elementary cells. The estimation does not take into account that not all the layers accept guest molecules (XRD spectra prove that $10 \%$ of interlayer distances in intercalate is the same as that in pure kaolinite). The correction leads to the conclusion that potassium acetate molecule falls, by average, into 1.7 elementary cells. It means that the expanded layers are only "supported" by the guest molecules between.

Following the conclusion concerning the location of the guest molecules, the intercalation should considerably alternate the positron annihilation spectrum. Table presents the positron annihilation experiments performed in pure kaolinite (KA) and in the intercalate with potassium acetate (KAPA). Each of the spectra was resolved into three components. Measurements were performed at room temperature, but the investigated samples were heated before the experiment to the demanded temperatures. For example, the notation KAPA-105 means that the intercalate kaolinite + potassium acetate was heated before the experiment up to $105^{\circ} \mathrm{C}$ (and then cooled to the room temperature in exsiccator). The demanded temperatures were selected on the basis of TG spectra. Reasonable qualities of fitting of the experimental decay curves were obtained when three processes were assumed. The first component (described by $\tau_{1}$ and the corresponding intensity $I_{1}$ ) is related to the direct annihilation in the Hostaphan ${ }^{\circledR}$ foil, inside the grains and annihilation of para-positronium. The second component $\tau_{2}$ (and the related intensity $I_{2}$ ) describes thermalized positronium trapping rate at the grains boundaries [9]. The last, long-lived component is related to the formation of $o$-Ps in large voids of low electron density.

When one compares the PALS results obtained in kaolinite and in intercalate (Table), the most spectacular is the considerable decrease of long-lived component in intercalated samples. Characteristic is also the intensity increase of the long-lived component after intercalation. XRD experiments presented above proved that the intercalation leads to a considerable increase in interlayer distances. However, the interlayer areas are not empty because guest molecules are located there. In natural kaolinite the space between gibbsite and silicate sheets are almost empty (except hydrogen bonds between the sheets). It means that the o-Ps appearing in this region can migrate along layers and its lifetime is relatively long. Unfortunately, the distance between sheets $(1.8 \AA)$ seems to be a limiting value available for the formation of positronium. Hence the probability of the formation of $o$-Ps (and adequate intensity) is low. In the case of intercalate, the distance between sheets increases considerably, but the interlayer area contains the guest molecules. The formation of $o$-Ps is more probable, but the pick-off process should shorten its lifetime. This explanation allows one to understand the observed shortening of long-lived component and the increase in intensity of this process. Heating both 
pure kaolinite and the intercalate decreases the long-living component. It could be related to the increase of imperfections in the material and to dehydroxylation appearing above $450^{\circ} \mathrm{C}$. In the case of intercalate, heating the sample much less influences positron annihilation parameters.

TABLE

Positron annihilation lifetimes, corresponding intensities, and dielectric permittivity for the examined samples.

\begin{tabular}{l|c|c|c|c|c|c|c|c}
\hline \multicolumn{1}{c|}{ Sample } & $T_{1}$ & $\mathrm{~d} T_{1}$ & $I_{1}$ & $\mathrm{~d} I_{1}$ & $T_{2}$ & $\mathrm{~d} T_{2}$ & $I_{2}$ & $\mathrm{~d} I_{2}$ \\
\hline KA-20 & 0.27 & 0.0028 & 79.13 & 2.57 & 0.47 & 0.02 & 19.14 & 2.48 \\
KA-150 & 0.25 & 0.0046 & 62.29 & 4.27 & 0.40 & 0.01 & 35.78 & 4.18 \\
KA-450 & 0.27 & 0.0026 & 64.38 & 1.59 & 0.52 & 0.01 & 33.49 & 1.49 \\
KAPA-20 & 0.17 & 0.01 & 26.04 & 2.79 & 0.36 & 0.01 & 44.90 & 2.45 \\
KAPA-105 & 0.16 & 0.02 & 22.80 & 2.00 & 0.35 & 0.01 & 47.60 & 1.70 \\
KAPA-250 & 0.15 & 0.04 & 17.60 & 2.40 & 0.34 & 0.01 & 61.70 & 2.10 \\
KAPA-450 & 0.24 & 0.01 & 48.70 & 4.50 & 0.44 & 0.03 & 37.70 & 4.40
\end{tabular}

TABLE continued

\begin{tabular}{l|c|c|c|c|c}
\hline \multicolumn{1}{c|}{ Sample } & $T_{3}$ & $\mathrm{~d} T_{3}$ & $I_{3}$ & $\mathrm{~d} I_{3}$ & $\varepsilon^{\prime}$ at $330 \mathrm{MHz}$ \\
\hline KA-20 & 8.15 & 0.33 & 1.73 & 0.12 & 6.64 \\
KA-150 & 5.60 & 0.18 & 1.93 & 0.13 & 6.01 \\
KA-450 & 4.28 & 0.20 & 2.13 & 0.13 & 4.88 \\
KAPA-20 & 1.18 & 0.12 & 29.06 & 0.52 & 4.25 \\
KAPA-105 & 1.14 & 0.05 & 29.57 & 1.22 & 3.17 \\
KAPA-250 & 1.42 & 0.09 & 20.66 & 1.58 & 3.93 \\
KAPA-450 & 1.24 & 0.09 & 13.61 & 1.74 & 3.28
\end{tabular}

The last property which we would like to discuss is a comparison of electric permittivity measured in kaolinite and in the intercalated material. Dielectric relaxation is sensitive about the existence of free volumes, if only polar molecules can rotate or librate under the influence of external electric field. The investigated materials are granular solids and the electric permittivity, obtained directly from measurement, is not the permittivity of kaolinite (or intercalate) but the permittivity of specific "conglomerate" of solid phase and air filling inter-granular free spaces. To obtain the "corrected" permittivity, the method of Robinson and Friedman [16] was used:

$$
\varepsilon_{s}=\frac{\varepsilon_{\mathrm{app}}-(1-\varphi) \varepsilon_{\mathrm{vac}}}{\varphi},
$$

where $\varepsilon_{s}$ is the corrected permittivity, $\varepsilon_{\text {app }}$ is the measured permittivity, $\varepsilon_{\mathrm{vac}}$ is the relative permittivity of vacuum (equal to 1 ), $\varphi$ is the volume fraction of the 
investigated material. The volume fraction of the material could be calculated as follows:

$$
\varphi=\frac{V(\text { solids })}{V(\text { sample })}=\frac{d(\text { sample })}{d(\text { solids })},
$$

where $d$ (sample) is density of the investigated sample, $d$ (solids) is density of the solids obtained on the base of crystallographic structure. The value of density $d$ (solids) estimated for pure kaolinite is $2.62 \mathrm{~g} / \mathrm{cm}^{3}$, and for the investigated intercalate $-1.76 \mathrm{~g} / \mathrm{cm}^{3}$.

Figure 2 shows the evolution of the real part of electric permittivity with frequency for materials heated to demanded temperatures. The imaginary part of dielectric permittivity is negligible and is not presented here. For kaolinite (the insetted picture) the electric permittivity was measured in samples heated to $150^{\circ} \mathrm{C}$ and $450^{\circ} \mathrm{C}$. The TG curve presented for kaolinite (Fig. 1) proves that up to $500^{\circ} \mathrm{C}$ the material was not decomposed. A small decrease in permittivity at the samples heated to $150^{\circ} \mathrm{C}$ (KA-150) and to $450^{\circ} \mathrm{C}$ (KA-450) is probably caused by removing the rest of water adsorbed at the surface and in the interlayer area.

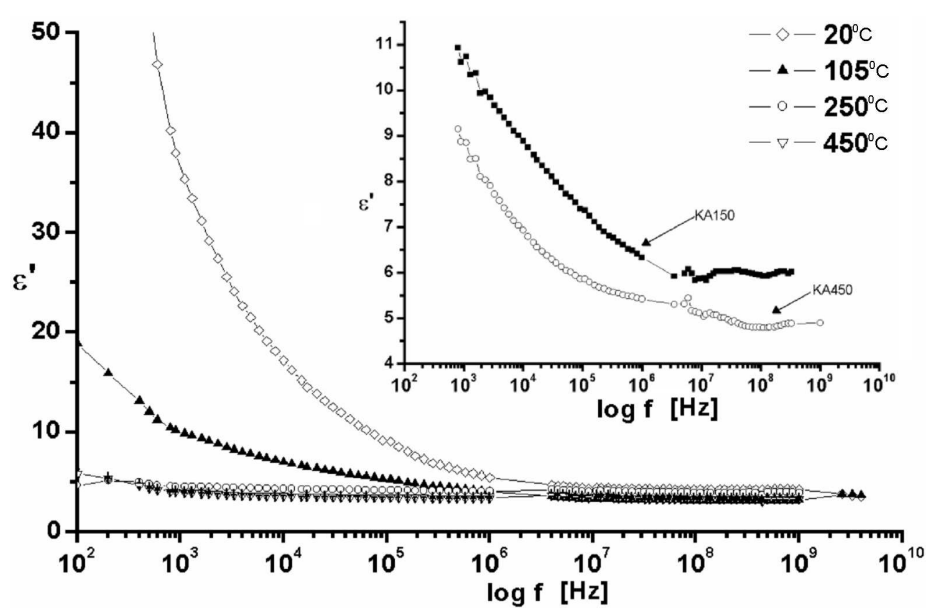

Fig. 2. Dielectric permittivity obtained for potassium acetate intercalate heated to demanded temperatures $\left(20,105,250,450^{\circ} \mathrm{C}\right)$. The insetted picture depicts permittivity measured for kaolinite heated to $150^{\circ} \mathrm{C}$ and $450^{\circ} \mathrm{C}$.

In low frequencies a little increase in $\varepsilon^{\prime}$ proves the influence of electrode polarization and/or the existence of spatial polarization of macroscopically inhomogeneous material. Figure 2 presents the $\varepsilon^{\prime}(f)$ dependence in the intercalate. It has to be stressed that the low frequency permittivity (measured below $10 \mathrm{kHz}$ ) is poorly repeatable. It strongly depends on the conditions of preparing of the sample and, especially, on humidity of atmosphere [17]. The intercalation by potassium acetate yields a highly hygroscopic product. It can be a general property. The intercalation leads to a considerable increase in interlayer distance and, hence, water 
molecules possibly will easily penetrate into the interlayer area. It has to result in the increase in the low-frequency permittivity and, hence, low repeatability of dielectric measurements (compare Orzechowski et al. [2]). Characteristic is the low-frequency increase obtained for the sample stored after preparation at room temperature over $\mathrm{P}_{2} \mathrm{O}_{5}$ in exsiccator (KAPA-20) and in the sample heated before the experiment to $105^{\circ} \mathrm{C}$ (KAPA-105). The increase could be related to slow movement of intercalated potassium acetate. However, in the samples heated to $250^{\circ} \mathrm{C}$ (KAPA-250) and $450^{\circ} \mathrm{C}$ (KAPA-450), the low frequency increase does not exist. It can be expected for the sample heated to $450^{\circ} \mathrm{C}$ because the intercalate is decomposed and kaolinite dehydroxylated. In contrary to that, the sample heated to $250^{\circ} \mathrm{C}$ is stable and if the low frequency increase in permittivity is related to the intercalated molecules, it should exist in KAPA-250 sample as well. Following that, the low frequency increase in the case of KAPA-150 could be rather a consequence of a little amount of water still remaining in the material. Interesting, but not fully understood property was observed at higher frequencies. As demonstrates the inset of Fig. 2, permittivity measured at $f>50 \mathrm{MHz}$ has an unexpected minimum of $\varepsilon^{\prime}$ for the sample denoted as KAPA-105. The decrease in permittivity means that some movements of polar groups are blocked. Cruz and Duro [18] presented XRD measurement performed in a function of temperature in kaolinite + potassium acetate intercalate. They observed that after heating to $60^{\circ} \mathrm{C}$ the peak responsible for the spacing $14 \AA$ disappears instead of two related to spacing $11.77 \AA$ and $9.3 \AA$. It means that heating a sample to only $105^{\circ} \mathrm{C}$ may change its structure and succeeding cooling to room temperature can yield a different material. Another possibility of the discussed decrease in permittivity could be a change of density of powdered material, not taken into account in calculations of the corrected permittivity (Eq. (2)).

\section{Conclusions}

Intercalation of clays leads to nanomaterials whose chemical and physical properties are not well recognized yet. In this paper we used XRD, thermal analysis, PALS, and dielectric relaxation measurements to characterize kaolinite intercalated by potassium acetate.

We found that the intercalation of kaolinite lowers the o-Ps lifetime and increases the contribution of the long-lived component in the global annihilation spectrum. This is because the interlayer distance increases, but the area is filled with potassium acetate which effects in increasing probability of pick-off process.

In spite of a considerable increase in free volumes, as detected by XRD and positron annihilation measurements, the intercalation of kaolinite by potassium acetate has a moderate influence on the electric properties of the material. In spite of a considerable increase in permittivity at low frequencies, linked rather with the content of water, the expected movement of guest molecules in the interlayer area is probably strongly hindered which shifts the expected relaxation towards lower 
frequencies. Electric permittivity of the material heated before experiments to $105^{\circ} \mathrm{C}$ was found lower than that in fresh sample or in samples heated to higher temperatures. This astonishing result can be linked with structural changes in the investigated material observed by other authors [5].

\section{Acknowledgments}

This work was supported by the State Committee for Scientific Research (Poland) the projects No. 3 T09B 05927 and No. 3 T09A 03129 as well as the grant of the Wrocław University No. 2016/IFD/06.

\section{References}

[1] Y. Deng, G.N. White, J.B. Dixon, J. Colloid Interface Sci. 250, 379 (2002).

[2] K. Orzechowski, T. Słonka, J. Głowiński, to be published in J. Phys. Chem. Solids.

[3] F. Stickel, T. Berger, R. Richert, E.W. Fisher, J. Chem. Phys. 107, 1086 (1997).

[4] M. Davies, K. Williams, Trans. Faraday Soc. 64, 529 (1968).

[5] Crystal Structures of Clay Minerals and Their X-ray Identification, Eds. G.W. Brindley, G. Brown, Mineralogical Society, London 1980.

[6] A. Weiss, W. Thielepape, W. Ritter, H. Schafer, G. Goring, Anorg. Allg. Chem. 320, 183 (1963).

[7] J.G. Thompson, C. Cuff, Clays and Clay Minerals 33, 400 (1985).

[8] G. Lagaly, Philos. Trans. R. Soc. Lond. A 311, 315 (1984).

[9] T.E.M. Staab, R. Krause-Rehberg, B. Kieback, J. Mater. Sci. 34, 3833 (1999).

[10] J.S. Nico, D.W. Gidley, A. Rich, Phys. Rev. Lett. 65, 1344 (1990).

[11] P. Kirkegaard, M. Eldrup, O. Mogensen, N.J. Pedersen, Comp. Phys. Commun. 23, 307 (1981).

[12] T. Goworek, K. Ciesielski, B. Jasińska, J. Wawryszczuk, Radiat. Phys. Chem. 58, 719 (2000).

[13] K. Wada, Am. Miner. 46, 78 (1961).

[14] M. Gabor, M. Toth, J. Kristof, K.H. Gabor, Clays and Clay Minerals 43, 223 (1995).

[15] L. Benco, D. Tunega, J. Hafner, H. Lischka, Am. Miner. 86, 1057 (2001).

[16] D.A. Robinson, S.P. Friedman, J. Non-Crystalline Solids 305, 261 (2002).

[17] J.C. Giuntini, A. Jabobker, J.V. Zanchetta, Clay Minerals 20, 347 (1985).

[18] M. Cruz, F. Duro, Clay Minerals 34, 565 (1999). 